\title{
Atomistic computer simulation of the clay-fluid interface in colloidal laponite
}

\author{
R. J. F. Leote de Carvalho and N. T. Skipper \\ Department of Physics and Astronomy, University College London, Gower Street, London WC1E 6BT, \\ United Kingdom
}

(Received 27 September 2000; accepted 4 December 2000)

\begin{abstract}
Monte Carlo and molecular dynamics computer simulations have been used to study the structure and dynamics of the interlayer aqueous solution in a colloidal sodium laponite clay at $277 \mathrm{~K}$. The system studied has a clay-clay spacing of $34.06 \AA$, and contains 1200 interlayer water molecules and 24 sodium counterions. The density profiles for interlayer species show two distinct layers of surface water as one moves away from the clay particles. The innermost of these layers is strongly oriented to form hydrogen bonds to the surface oxygen atoms. Radially averaged pair distributions have been calculated as a function of distance from the clay surfaces, and show that throughout our system the water structure is significantly perturbed from the bulk. In particular, we observe an increase in the second nearest-neighbor oxygen-oxygen distance, similar to that reported for low-density water at 268 K [A. K. Soper and M. A. Ricci, Phys. Rev. Lett. 84, 2881 (2000)]. The majority of the sodium counterions are fully hydrated by six water molecules. These hydrated ions have a strong tendency to remain close to the solid surfaces, as so-called "outer-sphere" complexes. However, we also observe cations further from the clay sheets, in the diffuse layer. Diffusion of water and cations in the plane of the clay sheets is comparable to that in the bulk, but is significantly reduced normal to the clay sheets. (C) 2001 American Institute of Physics.
\end{abstract}

[DOI: $10.1063 / 1.1343839]$

\section{INTRODUCTION}

Molecular level understanding of the properties of aqueous solutions close to charged solid surfaces, in the electrical double-layer, is of fundamental importance for many processes. These include colloidal interactions, membrane formation and stability, protein folding, electrode reactions, and mineral formation and dissolution. The conventional picture of the electrical double-layer region is based on the primitive model, in which the solvent is treated as a dielectric continuum. ${ }^{1,2}$ Electrostatic interactions among the ions and surfaces then give rise to so-called condensed (Stern) and diffuse (Gouy-Chapman) ion distributions. However, recent experiments have shown that for surface-surface separations less than $30 \AA$ the molecular nature of water and the hydrated species dominates the structure and interactions. ${ }^{3-7}$ In this regime atomistic level computer simulations can play a very valuable role by providing detailed portraits of the interfacial aqueous phase.

Computer studies of aqueous solutions at charged metallic surfaces have already made significant contributions to our understanding of electrochemical systems..$^{2,4,8-11}$ In addition, work on hydrated clays has resolved many key problems concerning hydration and speciation at charged silicate surfaces. ${ }^{12-14}$ However, for practical reasons, most of this previous research has focused on surfaces separated by only a few molecular layers of water. There is now an opportunity to extend this approach to systems at the limit of validity for primitive models.

In this paper we report on computer simulations of hydrated sodium laponite clay with a clay layer spacing in ex- cess of $30 \AA$, using models and methodology developed during previous studies of lower clay hydrates. We analyze the interlayer structure and diffusion parallel and perpendicular to the clay sheets. In addition, we calculate the density of interlayer water as a function of distance from the clay sheets. We observe two distinct layers of surface water as one moves away from the clay particles. However, structural analysis shows that throughout our system water is significantly perturbed from the bulk. Cations are predominantly present as fully hydrated "outer-sphere", complexes, ${ }^{15}$ located approximately $4.25 \AA$ from the nearest clay surface.

\section{INTERACTION POTENTIALS AND METHODOLOGY}

\section{A. Simulation box}

The simulation box contains a 42.24 by $36.56 \AA$ slab of laponite clay, 24 sodium counterions, and 1200 interlayer water molecules (Fig. 1). The overall equilibrium clay-clay spacing is $34.06 \pm 0.08 \AA$. Periodic boundary conditions replicate the simulation box in three dimensions, creating a stack of infinite parallel clay layers with water and sodium counterions free to move in the interlayer space.

Laponite is a synthetic smectite clay that resembles the natural clay hectorite in structure and composition. ${ }^{16}$ Atomic positions and charges of the unit cell used to create our slab of laponite are given in Table I. This cell was replicated through a point of inversion at the origin, and converted to a rhombohedral unit. The composition of this unit is on average

$$
\left[\mathrm{Mg}_{5.25} \mathrm{Li}_{0.75}\right] \mathrm{Si}_{8} \mathrm{O}_{20}(\mathrm{OH})_{4} \mathrm{Na}_{0.75} \text {. }
$$




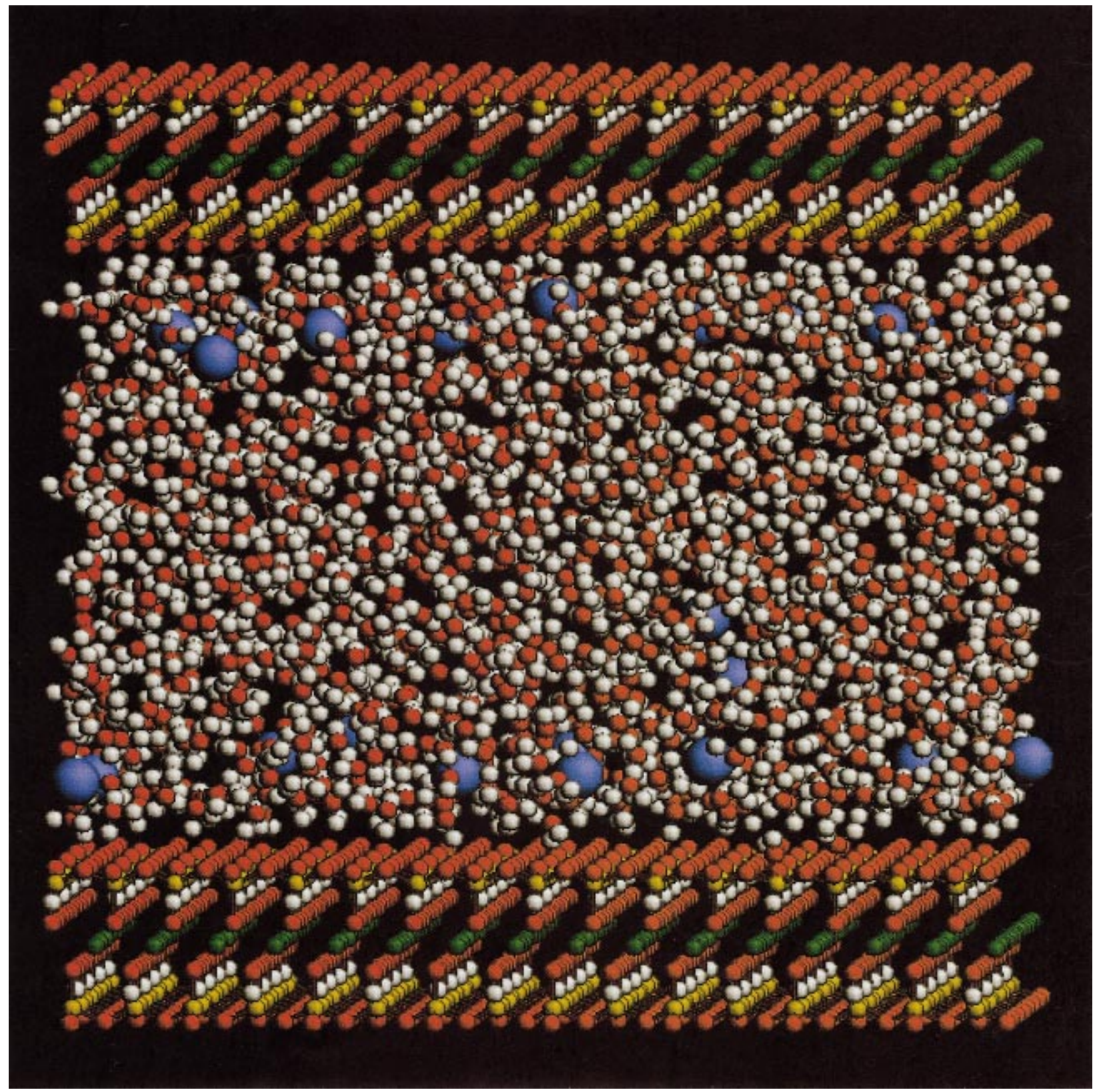

FIG. 1. (Color) Snapshot of the molecular dynamics simulation box used for the current studies of the interlayer fluid structure and dynamics in laponite. Each laponite layer contains a total of 1344 atoms and possesses a net negative charge of $-24 e$. The layer on top was added for better visualization. The dimensions of the cell are 42.24, 36.56, and $34.06 \AA$ in $x$-, $y$-, and $z$-directions, respectively. The interlayer region contains 1200 water molecules and 24 charge balancing sodium counterions. Oxygen atoms are represented by red spheres and hydrogen by the white. The larger blue spheres are sodium cations. The sheet in the middle of each clay layer contains green lithium and magnesium. The remaining yellow atoms, bonded to oxygen atoms on the surface of the clay layer, are silicon.

It was then repeated 32 times, 8 times along the $x$-direction and 4 times along the $y$-direction. The clay sheet in the simulation box therefore contains a total of 1344 atom sites. The overall negative charge arises from substitution of lithium for magnesium among the octahedrally coordinated cations that are located at the midplane of the clay sheets. These sites were selected to lie as far apart as possible, thereby producing a regular rather than random array of lithium atoms. The overall charge of the simulation slab is $-24 e$, so the charge density is $0.24 \mathrm{~cm}^{-2}$.

\section{B. Interaction potentials}

The interaction potentials used here are the ones of Boek et al. ${ }^{17}$ These are based on the TIP4P model of water ${ }^{18-20}$ which represents water as a rigid molecule with four interaction sites. Site-site interactions involve Coulomb and Lennard-Jones 6-12 terms,

$$
v_{i j}\left(r_{i j}\right)=\frac{q_{i} q_{j}}{r_{i j}}-\frac{A_{i j}}{r_{i j}^{6}}+\frac{B_{i j}}{r_{i j}^{12}} .
$$

A charge $+0.52 e$ is placed on each of the hydrogen sites and a negative charge $-1.04 e$ on a massless site, situated towards the hydrogen atoms and along $C_{2}$ symmetry axis of the water molecule. The fourth site is a neutral oxygen atom, which is the only Lennard-Jones site on the water molecule. The parameters for the $\mathrm{O}-\mathrm{O}$ interaction are shown in Table II. The distances between the massless site, $M$, the hydrogen 
TABLE I. Atomic positions and respective effective local charges in the unit cell of laponite. The local charges shown were used in Eq. (2) for the pair interactions. ${ }^{a}$ In the simulation cell shown in Fig. 1, one in four octahedral $\mathrm{Mg}$ sites are replaced by $\mathrm{Li}$, with a charge of $+1.00 e$.

\begin{tabular}{lrrrr}
\hline \hline Atom & $x(\AA)$ & $y(\AA)$ & $z(\AA)$ & $q(e)$ \\
\hline O (surface) & 2.64 & 0.00 & 3.28 & -0.80 \\
O (surface) & 1.32 & 2.28 & 3.28 & -0.80 \\
O (surface) & 3.96 & 2.28 & 3.28 & -0.80 \\
$\mathrm{O}(\mathrm{OH})$ & 0.00 & 0.00 & 1.06 & -1.52 \\
$\mathrm{H}(\mathrm{OH})$ & 0.00 & 0.00 & 2.0176 & +0.52 \\
$\mathrm{Si}$ & 2.64 & 1.52 & 2.73 & +1.20 \\
$\mathrm{Si}$ & 5.28 & 3.05 & 2.73 & +1.20 \\
$\mathrm{O}$ (apical) & 2.64 & 1.52 & 1.06 & -1.00 \\
$\mathrm{O}$ (apical) & 5.28 & 3.05 & 1.06 & -1.00 \\
$\mathrm{Mg}$ & 1.76 & 0.00 & 0.00 & +2.00 \\
$\mathrm{Mg}$ & -0.88 & 1.52 & 0.00 & +2.00 \\
$\mathrm{Mg}$ & -0.88 & -1.52 & 0.00 & +2.00 \\
\hline \hline
\end{tabular}

${ }^{\text {a Reference } 17 .}$

atoms, and the oxygen are $r_{\mathrm{MO}}=0.15 \AA$ and $r_{\mathrm{HO}}=0.9572 \AA$, respectively. The angle $\widehat{\mathrm{HOH}}$ is fixed at $104.52^{\circ}$.

The water-sodium interactions used in the simulations are the ones incorporated into the "OPLS" set of interaction potentials, ${ }^{19}$ and are compatible with the TIP4P model. ${ }^{17}$ The clay sheets were then adapted to the TIP4P-OPLS models by placing interaction sites on the oxygen atoms, with LennardJones parameters equal to those in TIP4P water. Partial charges are assigned to clay sites as shown in Table I.

\section{Simulation runs}

The starting configuration was prepared by placing sodium counterions $8 \AA$ from the lithium sites in the clay sheet. Water molecules were placed randomly in the remaining interlayer space, but at least $1.5 \AA$ from the nearest site. The starting clay layer spacing was $34 \AA$. A constant stress Monte Carlo preequilbration run of $10^{6}$ iterations was then conducted at $277 \mathrm{~K}$ and with a pressure of $1 \times 10^{5} \mathrm{~Pa}$ applied normal to the clay sheets (in the $z$-direction). ${ }^{21}$ Equilibration was conceded when total energy and clay layer spacing oscillated about their mean values in the normal way. The equilibrated system was then passed on for molecular dynamics.

Molecular dynamics simulations were conducted using the code MOLDY. ${ }^{22}$ We used a time step of $0.0005 \mathrm{ps}$, and allowed the system to equilibrate for a further $5 \times 10^{4}$ time steps. During equilibration, a Nosé-Hoover thermostat was coupled to the system. For this purpose the temperature/ velocities were rescaled every 250 time steps, using the instantaneous kinetic energy, for each species individually.

TABLE II. Lennard-Jones parameters used in Eq. (2) for pair interactions. ${ }^{a}$

\begin{tabular}{lcc}
\hline \hline & $A\left(\mathrm{kcal} \AA^{6} \mathrm{~mol}^{-1}\right)$ & $B \times 10^{-3}\left(\mathrm{kcal} \AA^{12} \mathrm{~mol}^{-1}\right)$ \\
\hline $\mathrm{O}-\mathrm{O}$ & 610 & 600 \\
$\mathrm{O}-\mathrm{Na}^{+}$ & 428 & 91.7 \\
$\mathrm{Na}^{+}-\mathrm{Na}^{+}$ & 300 & 14.0 \\
\hline \hline
\end{tabular}

${ }^{\mathrm{a} R e f e r e n c e ~} 17$.
During this part of the simulation we again applied a uniaxial stress $1 \times 10^{5} \mathrm{~Pa}$ perpendicular to the clay sheets, but no change in $\bar{z}$ was noted.

During the production molecular dynamics run the system sampled the $(N, V, E)$ ensemble. In total, we present results from $2 \times 10^{5}$ time steps of $0.0005 \mathrm{ps}$, in other words 100 ps. No velocity rescaling was required during this period. The structure of the interlayer fluid was recorded every 100 time steps, from which we obtained average samples.

Long-range Coulomb interactions were handled using the Ewald sum technique in three dimensions. ${ }^{22}$ The parameters used in the Ewald sum were optimized by MOLDY: the reciprocal space cutoff was set to $1.82 \AA^{-1}$ and the real/ reciprocal partition parameter, $\alpha=0.268 \AA^{-1}$. An interaction cutoff of $12.64 \AA$ was used. MOLDY does not follow the minimum image convention. Instead, the list of neighboring cells includes all periodic images of a particle which are within the interaction cutoff.

In order to calculate the nominal volume occupied by the interlayer fluid, a control simulation of dry laponite was carried out by simply removing all water molecules from the system. A Monte Carlo simulation at constant $p=10^{5} \mathrm{~Pa}$ and temperature $T=277 \mathrm{~K}$ gave a dry clay-clay spacing of 9.50 $\AA$, and an equilibrium volume $V(0)=14670.8 \AA^{3}$ for the simulation box, which compares to $V(1200)=52598.7 \AA^{3}$ for the hydrated laponite. The apparent average density of water in our laponite can then be calculated using the effective volume $V(1200)-V(0)$. This procedure gives 0.95 $\pm 0.02 \mathrm{~g} \mathrm{~cm}^{-3}$ for the interlayer water. However, we note here that the simulated spacing for dry clay is rather low, due to the ability of the sodium counterions to sink into the hexagonal ring sites on the clay surface. If we take the experimental value of $10.28 \AA$ for a dry clay of low tetrahedral charge, ${ }^{23}$ the water density is then $0.98 \pm 0.02 \mathrm{~g} / \mathrm{cm}^{-3}$.

A molecular dynamics simulation of bulk water at $T$ $=277 \mathrm{~K}$ and $p=10^{5} \mathrm{~Pa}$ was also performed with 256 water molecules interacting via the TIP4P potential. The system was first allowed to relax into the appropriate volume, leading to an equilibrium density $\rho_{\text {water }}=1.02 \pm 0.01 \mathrm{~g} \mathrm{~cm}^{-3}{ }^{20}$ The equilibration was followed by a production run of 100 ps, again $2 \times 10^{5}$ time steps of 0.0005 ps. This allowed us to extract structural and dynamical information for comparison.

\section{RESULTS AND DISCUSSION}

We present results for the structure and dynamics of the interlayer solution, both parallel and normal to the plane of the clay sheets. Data are presented for single particle density profiles $\left[\rho_{\alpha}(x), \rho_{\alpha}(y)\right.$ and $\left.\rho_{\alpha}(z)\right]$, and self-diffusion coefficients $\left(D_{\alpha}\right)$. In addition, we calculate pair distribution functions for interlayer species $\left[g_{\alpha \beta}(r)\right]$, as a function of a particle's distance from the clay surfaces.

\section{A. Density profiles}

The density profiles normal to the clay sheets were calculated from the 2000 interlayer configurations dumped during the production run, using the equation

$$
\rho_{\alpha}(z)=\frac{1}{A_{x y}} \frac{d n_{\alpha}(z)}{d z},
$$




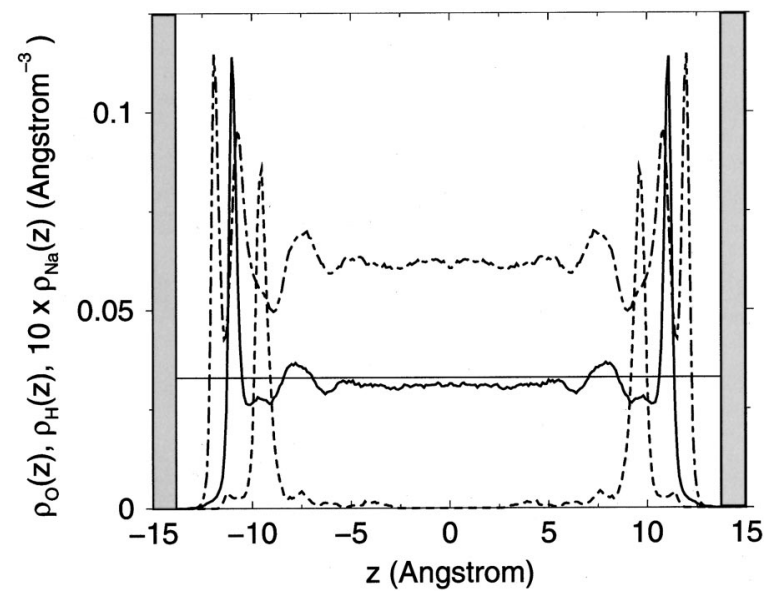

FIG. 2. Density profiles normal to the clay sheets: $\rho_{\mathrm{O}}(z)$ for oxygen (solid curve), $\rho_{\mathrm{H}}(z)$ for hydrogen (dotted-dashed curve), and $\rho_{\mathrm{Na}}(z)$ for the sodium counterions (dashed line). The latter has been multiplied by a factor of 10 for better visualization. The horizontal line shows the density of oxygen atoms in pure bulk TIP4P water at $277 \mathrm{~K}$.

with $\alpha=\mathrm{O}, \mathrm{H}$, and $\mathrm{Na}^{+} . A_{x y}=1544.29 \AA^{2}$ is the area of the simulation box in the $x y$-plane and $d n_{\alpha}(z)$ the number of atoms of species $\alpha$ between planes $z$ and $z+d z$. The density profiles for the interlayer oxygen and hydrogen and sodium cations, $\rho_{\mathrm{O}}(z), \rho_{\mathrm{H}}(z)$, and $\rho_{\mathrm{Na}}(z)$, respectively, are plotted in Fig. 2. The latter was magnified by a factor of 10 for better visualization. The $z$-axis on these figures represents the distance to the midplane between two parallel opposite clay layers. The surface oxygens of the clay sheets therefore lie at $\pm 13.75 \AA$.

The density profile $\rho_{\mathrm{H}}(z)$ exhibits two large peaks near the clay surface, at $|z|=11.9$ and $10.7 \AA$. The $\rho_{\mathrm{O}}(z)$ shows one large peak, also near the clay surface, at $|z|=11.0 \AA$, between the two large hydrogen peaks. A smaller oxygen peak occurs at $|z|=7.5 \AA$, and is coincident with a similar feature in $\rho_{\mathrm{H}}(z)$. We therefore conclude that the first layer of water is hydrogen-bonded to the surface oxygen atoms of the clay surface. The second molecular layer of water is not strongly oriented with respect to the clay sheets, and therefore acts as an intermediary between the surface and the bulk. This picture is consistent with those derived from previous simulations of clay systems, ${ }^{12-14}$ and negatively charged metal surfaces. $^{2,4,8-11}$

The sodium density profile $\rho_{\mathrm{Na}}(z)$ has a dominant peak at $|z|=9.5 \AA$, a distance of $4.25 \AA$ from the clay surface. These cations are fully hydrated by six water molecules (see Sec. III B), and are termed outer-sphere complexes. ${ }^{15}$ In spite of their proximity to the clay surface, these cations are not bound to any particular lithium site, as we will see from the diffusion coefficients calculated in Sec. III C. A relatively small fraction of sodiums exist as partially hydrated "innersphere" complexes, in direct contact with the clay surface and directly above a lithium atom. Further from the clay sheets we see the beginnings of a diffuse population of counterions. The distribution in this region is still weakly discretized, presumably by the formation of second and third hydration spheres. This scenario suggests that the surface of laponite is covered almost exclusively by adsorbed water molecules.

The time scale of the simulations and relatively small number of counterions prevents us from drawing unambiguous conclusions regarding the relative populations of peaks in $\rho_{\mathrm{Na}}(z)$. However, it should be born in mind that the starting point of the sodium counterions was $8 \AA$ from the nearest clay sheets, and so the ions therefore had to travel towards the clay sheet to reach the main peak at $|z|=9.5 \AA$. During the 100 ps of simulation no sodium cation traveled across the simulation box, from one surface layer to the opposite side.

There are very few direct measurements of aqueous structure at a colloidal surface. Most relevant to our current work are high resolution neutron diffraction studies of hydrated vermiculite clays, using isotopic labeling. ${ }^{5,6}$ In these studies the counterions were short chain alkylammonium, such as $\mathrm{C}_{3} \mathrm{H}_{7} \mathrm{NH}_{3}^{+}$, with clay-clay spacings of the order 40 $\AA$. Two ordered layers of water are indeed observed close to the clay sheets, but the counterion distribution is centered on the midplane of the interlayer region. The latter may be a peculiarity of the particular counterions, which have hydrophobic end-groups, or the clay surface, which is more highly charged than laponite. ${ }^{16}$ Surface force measurements of interactions between hydrated mica clay plates show oscillations at separations of less than $30 \AA^{3}{ }^{3}$ These are consistent with our calculated layering of water at the surface, but could equally well arise from the different counterion hydration states observed in our simulations. X-ray standing wave studies of the rutile-water interface provide quite detailed information on the counterion distribution. ${ }^{7}$ Recent studies on rubidium ions show a large population in the innersphere, probably due to the very weak hydration of this cation. $^{24}$

For us to understand the thermodynamics of the confined water, it is important to establish the interlayer density. We have already shown that the average value for water in our laponite is slightly lower than that in the bulk $(0.98 \pm 0.02$ and $1.02 \pm 0.02 \mathrm{~g} / \mathrm{cm}^{-3}$, respectively). However, this comparison depends on the (arbitrary) definition of the dry clay volume. For this reason it is instructive for us to calculate the water density as a profile across the interlayer region. To aid the reader, the number density of oxygen atoms in bulk water, $0.0335 \AA^{-3}$, is shown as the solid horizontal line in Fig. 2. It can be seen that even towards the center of the interlayer region, this asymptote exceeds the density of the confined water. In fact, the mid-plane interlayer figure of $0.0310 \AA^{-3}$ is closer to the value of $0.0295 \AA^{-3}$ obtained for low density water at $268 \mathrm{~K} .{ }^{25}$ We discuss the structural interpretation of this effect in Sec. III B.

Density profiles in the plane of the clay sheets were also computed for water molecules adsorbed to the clay surface using, for $x$ for example,

$$
\rho_{\alpha}(x)=\frac{1}{A_{y z}} \frac{d n_{\alpha}(x)}{d x} .
$$

These data are shown in Fig. 3. The cutoff for the first layer of water molecules was taken as $|z|>10.0 \AA$ from the midplane of the interlayer region. The position of oxygen and lithium atoms in the clay sheet is indicated in these figures, 

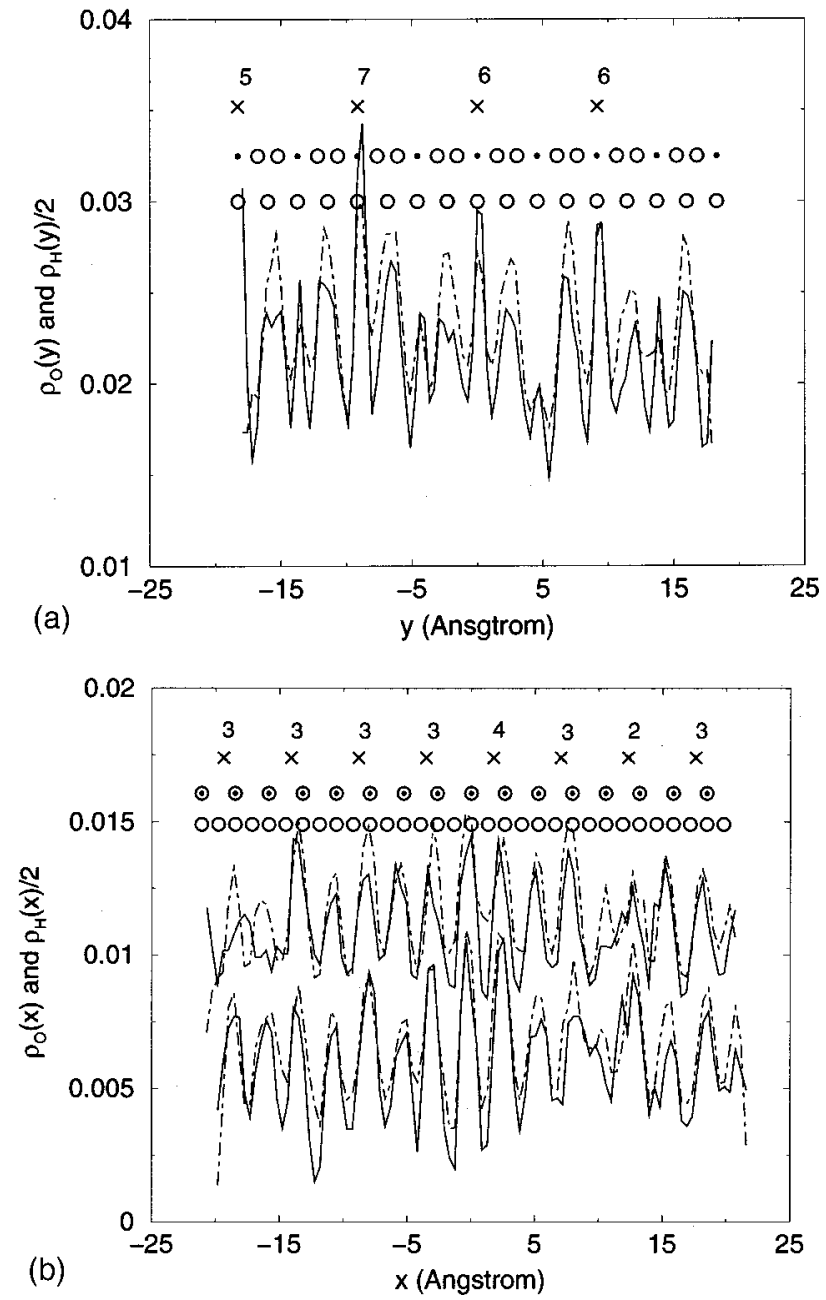

FIG. 3. Density profiles parallel to the clay sheets. In (a), the density profiles for interlayer oxygen $\rho_{\mathrm{O}}(y)$ (solid curve) and hydrogen $\rho_{\mathrm{H}}(y)$ (dotteddashed curve). In (b), $\rho_{\mathrm{O}}(x)$ (solid curve) and $\rho_{\mathrm{H}}(x)$ (dotted-dashed curve). These were calculated for oxygen and hydrogen in the first adsorbed layer of interlayer water molecules, for which $|z|>10.0 \AA$. The $\rho_{\mathrm{H}}$ were halved for easier visualization. Due to the symmetry of the crystallographic cell of laponite, in (a) the density profiles were calculated adding the top and the bottom surface profiles whereas in (b) the calculation was done separately for the top (curves on top) and bottom surfaces (curves below, shifted downwards by 0.005 for better visualization) of the clay layer. The positions of the rows containing surface and apical oxygen atoms in the clay layer are represented with open circles. The positions of rows containing oxygen atoms in the clay $\mathrm{OH}$ groups are represented with dots. The positions of rows of magnesium with lithium are marked with crosses. The number of vacancies on each row is indicated.

and for better visualization the magnitude of the hydrogen profiles were halved. Due to the offset of the upper and lower surface oxygen atoms when viewed in the $y z$-plane, the profiles $\rho_{\mathrm{O}}(x)$ and $\rho_{\mathrm{H}}(x)$ on the top and bottom surfaces of the clay layer were plotted separately in Fig. 3(b), with those for the bottom layer shifted to register with the upper clay atoms.

The in-plane profiles show that hydrogen and oxygen maxima coincide, confirming strong hydrogen bonding to every other surface oxygen atom on the clay surface. The separation between these atoms is $2.64 \AA$. The remaining hydrogen atoms are not registered to the clay surface. It is also interesting to note that the highest hydrogen peaks occur

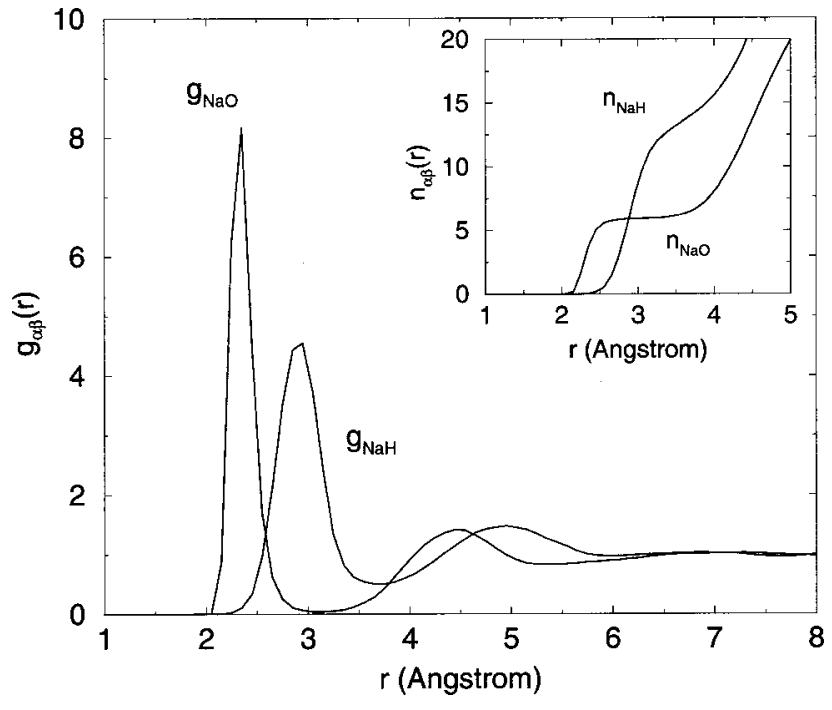

FIG. 4. Sodium-centered pair distribution functions, $g_{\mathrm{NaH}}(r)$ and $g_{\mathrm{NaO}}(r)$, averaged over the whole simulation box. The coordination numbers $n_{\alpha \beta}(r)$ are represented in the insets, and show sixfold hydration.

near positions of the magnesium row containing the highest number of lithium substitutions, and therefore the highest negative charge density. The results in Fig. 3 therefore clearly indicate the high level of organization of the first adsorption layer of water on the surface of laponite.

\section{B. Pair distribution functions}

The three-dimensionally averaged radial pair distribution functions are plotted in Figs. 4 and 5 and were computed from the simulation data using the definition

$$
g_{\alpha \beta}(r)=\frac{1}{4 \pi \rho_{\beta} r^{2}} \frac{d n_{\alpha \beta}(r)}{d r},
$$

where $d n_{\alpha \beta}(r)$ is the number of particles of species $\beta$ (in the interlayer region and clay) at a distance between $r$ and $r$ $+d r$ of a fixed particle of interlayer particles of species $\alpha$. The function $n_{\alpha \beta}(r)$ is also known as the running coordination number and is plotted as insets in Figs. 4 and 5.

Figure 4 shows the sodium-centered distribution functions $g_{\mathrm{NaH}}(r)$ and $g_{\mathrm{NaO}}(r)$, with the respective coordination numbers in the inset. The first peaks in $g_{\mathrm{NaO}}(r)$ and $g_{\mathrm{NaH}}(r)$ occur at 2.4 and $3.0 \AA$, respectively, and give a coordination number of six water molecules when $r<3.5 \AA$. This is very similar to the situation found in bulk water, ${ }^{24}$ and confirms that "correct" hydration of the counterions is a priority for the interlayer water.

Turning now to the water itself, the large distance between our two opposing layers of laponite makes it is useful for us to resolve the $g_{\alpha \beta}(r)$ into contributions from particles of species $\alpha$ lying within a certain distance of the clay surface. In Fig. 5 we chose four windows of range $z_{0}-2.13 \AA$ $<|z|<z_{0}+2.13 \AA$. The dotted-dashed lines include particles closest to the clay, with $z_{0}=12.78 \AA$. The long-dashed line was computed for $z_{0}=8.52 \AA$, the dashed line corresponds to $z_{0}=4.26 \AA$, and the dotted line to $z_{0}=0.0 \AA$. In the insets of Fig. 5 the corresponding coordination numbers are plotted. For reference, the distribution functions for bulk water are 

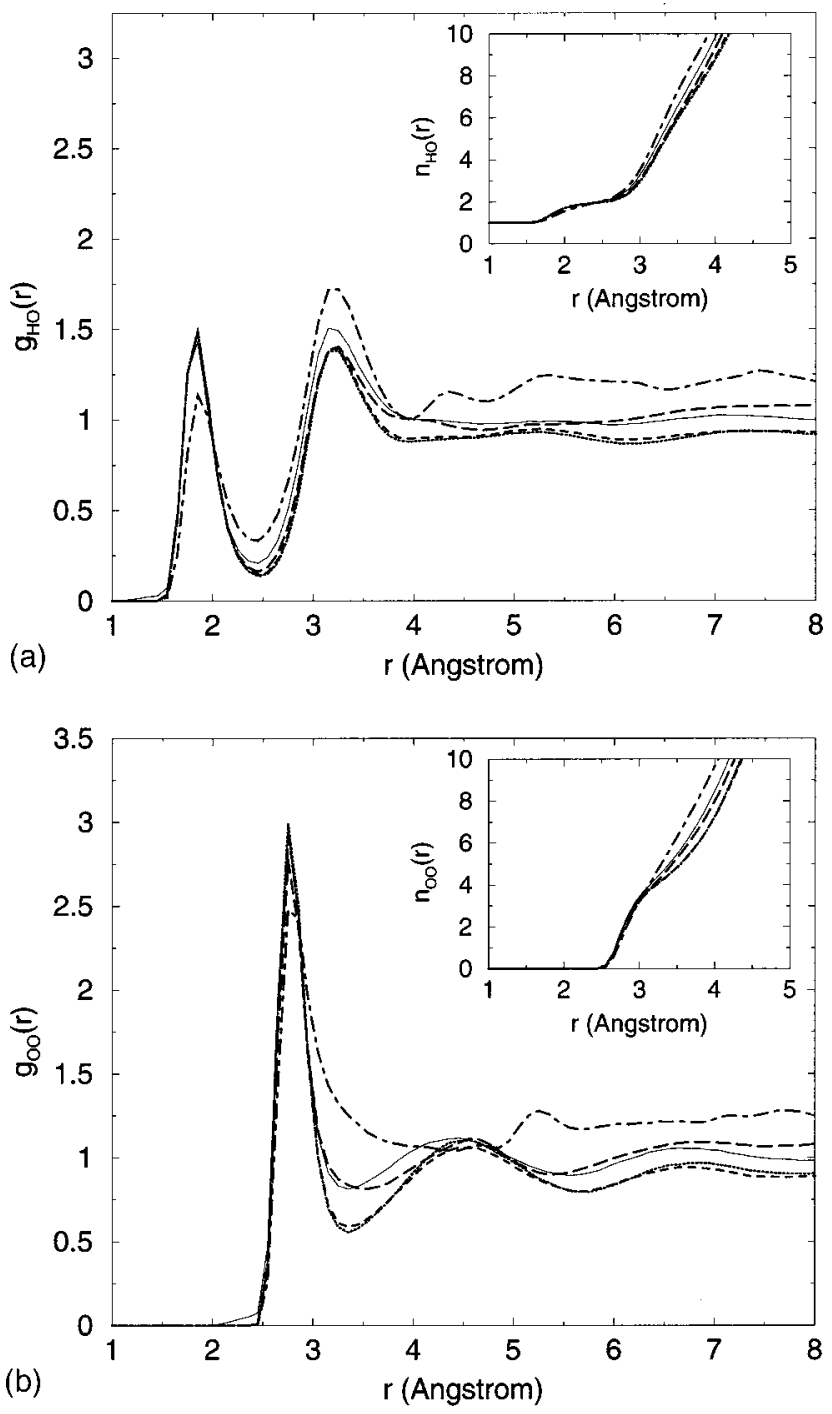

FIG. 5. Pair distribution functions for interlayer species, resolved into distance from the midplane of the clay sheets. In (a) distribution functions $g_{\mathrm{HO}}(r)$ and in (b) $g_{\mathrm{OO}}(r)$ for interlayer water in laponite. These $g_{\alpha \beta}(r)$ were calculated by counting the $\beta$ atoms (interlayer and clay atoms) lying at a distance $r$ from a fixed interlayer $\alpha$ atom which is within a distance $z_{0}$ $-2.13 \AA<|z|<z_{0}+2.13 \AA$ from the central magnesium $x y$-plane in the clay sheet. The different values $z_{0}$ are $12.78 \AA$ (dotted-dashed line), $8.52 \AA$ (long-dashed line), $4.26 \AA$ (dashed line), and $0.0 \AA$ (dotted line). The distribution functions for bulk water (solid lines) are also plotted for comparison. All corresponding coordination numbers $n_{\alpha \beta}(r)$ are shown in the insets.

also presented as the solid lines. These data show that nearest-neighbor hydrogen bonding is honored for all but the first molecular layers of water. This is consistent with the picture derived from neutron diffraction studies of interlayer water in smectite clay. ${ }^{26}$ However, even among molecules over $10 \AA$ from the nearest clay surface, longer range structure has still not recovered to the bulk. In particular, we observe a shift to higher $r$ for the second nearest-neighbor $\mathrm{O}-\mathrm{O}$ correlations occurring at around $4.5 \AA$. The imposition of planar geometry therefore produces a more open water structure, similar to that proposed for low-density water in the bulk at $268 \mathrm{~K} .{ }^{25}$ Note also that the running coordination numbers, $n(r)$, for water outside the surface layer are below the bulk values. This correlates the reduced local density to the observed structural changes. For this reason, our simula-
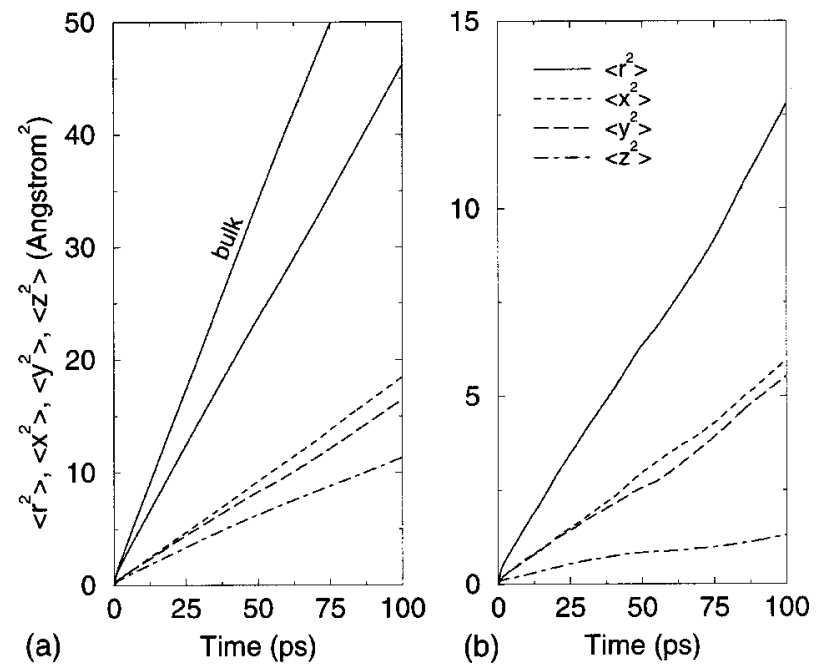

FIG. 6. The total mean-square displacement $\left\langle r^{2}\right\rangle$ and directional meansquare displacements $\left\langle x^{2}\right\rangle,\left\langle y^{2}\right\rangle$, and $\left\langle z^{2}\right\rangle$ for (a) the interlayer water and (b) the sodium counterions, in laponite, as a function of simulation time. In (a), the $\left\langle r^{2}\right\rangle$ for bulk water is also shown.

tions lead us to conclude that the clay-water interface is longer range than the oscillations in the density profiles of Fig. 2.

\section{Diffusion properties}

The self-diffusion coefficients $D_{\alpha}$ for the different species can be computed from the mean-square displacement

$$
\left\langle r^{2}\right\rangle \equiv\left\langle\left|\mathbf{r}(t)-\mathbf{r}\left(t_{0}\right)\right|^{2}\right\rangle \approx \frac{1}{N N_{t}} \sum_{n=t}^{N} \sum_{t_{0}}^{N_{t}}\left|\mathbf{r}_{n}(t)-\mathbf{r}_{n}\left(t_{0}\right)\right|^{2},
$$

where $\mathbf{r}_{n}(t)$ is the position of particle $n$ at time $t, N$ is the number of particles, and $N_{t}$ is the number of time steps. The Einstein diffusion equation relates $D$ to the dimensionality $d_{0}$ and to the gradient of $\left\langle r^{2}\right\rangle$ with time

$$
D=\frac{1}{2 d_{0}} \frac{d\left\langle r^{2}\right\rangle}{d t} \text {. }
$$

The mean-square displacement for interlayer water and sodium counterions was computed from the simulation data and are plotted in Fig. 6. We present the total threedimensional $\left\langle r^{2}\right\rangle$, as well as the individual one-dimensional $\left\langle x^{2}\right\rangle,\left\langle y^{2}\right\rangle$, and $\left\langle z^{2}\right\rangle$. The self-diffusion coefficients were computed from these plots and are listed in Table III. The

TABLE III. Self-diffusion coefficients of interlayer water molecules and sodium counterions in laponite at $T=277 \mathrm{~K}$ and $p=10^{5} \mathrm{~Pa}$.

\begin{tabular}{lcc}
\hline \hline & Water & Sodium \\
\hline$D_{\text {total }} \times 10^{10}\left(\mathrm{~m}^{2} \mathrm{~s}^{-1}\right)$ & 7.4 & 2.0 \\
$D_{x} \times 10^{10}\left(\mathrm{~m}^{2} \mathrm{~s}^{-1}\right)$ & 9.2 & 2.9 \\
$D_{y} \times 10^{10}\left(\mathrm{~m}^{2} \mathrm{~s}^{-1}\right)$ & 8.1 & 2.7 \\
$D_{z} \times 10^{10}\left(\mathrm{~m}^{2} \mathrm{~s}^{-1}\right)$ & 4.9 & 0.48 \\
Bulk water & $D_{\text {water }}=1.1 \times 10^{-9} \mathrm{~m}^{2} \mathrm{~s}^{-1}$ & \\
Infinite dilution $(297 \mathrm{~K})^{\mathrm{a}}$ & $D_{\mathrm{Na}}=1.0 \times 10^{-9} \mathrm{~m}^{2} \mathrm{~s}^{-1}$ & \\
\hline
\end{tabular}

${ }^{\mathrm{a}}$ Reference 24 . 
coefficient for the self-diffusion of water in bulk is also given in this table, and was calculated from the separate simulation with 256 water molecules at the same temperature and pressure.

The results in Fig. 6(a) show that the diffusion of water in our laponite is anisotropic, with greatest mobility parallel to the clay sheets. In fact, in-plane diffusion approaches that of the bulk. By bearing in mind that the first two molecular layers of water are severely hampered by the clay sheets, ${ }^{27}$ we conclude that around the midplane of our system, water molecules are at least as mobile as they are in the bulk. Unfortunately it is not possible for us to resolve diffusion into slices similar to those used for the radial distribution functions, because of the motion normal to the clay sheets.

The sodium counterions are predominantly present as outer-sphere hydrated complexes, and their motion is therefore strongly influenced by the clay surface. For this reason also, the in-plane diffusion coefficients of the counterions are comparable to those observed in clay systems containing only three molecular layers of water. ${ }^{27}$

Finally we note that the diffusion of interlayer sodium (and water) is not perfectly equivalent in the $x$ - and $y$-directions along the surface: both water and sodium cations diffuse slightly faster along our chosen $x$-direction. Figure 3 shows that not only is the density of surface siloxane oxygens higher along the $x$-direction, but also that the number of lithium defects is slightly lower along this direction. These structural asymmetries are responsible for the slightly higher diffusion of sodium ions, and the water hydrated to them, along the $x$-direction. The fact that this effect can be observed is a consequence of the relatively large scale of the simulation.

\section{CONCLUSIONS}

Monte Carlo and molecular dynamics computer simulation has been used to study the structure and dynamics of an interlayer aqueous solution in a colloidal sodium laponite clay. The system studied has a clay-clay spacing of $34.06 \AA$, and contains 1200 interlayer water molecules and 24 sodium counterions. The density profiles for interlayer species show two distinct layers of surface water as one moves away from each clay particle. The innermost of these layers is strongly oriented to form hydrogen bonds to the surface oxygen atoms. Radially averaged pair distributions, calculated as a function of distance from the clay surfaces, show that throughout our system the water structure is significantly perturbed from the bulk. In particular, we observe a shift to higher $r$ for the second nearest-neighbor $\mathrm{O}-\mathrm{O}$ correlations occurring at around $4.5 \AA$. This structural change can be correlated to a reduction in the local density, visible in the running coordination numbers $n(r)$. The imposition of planar geometry therefore leads to a more open water structure, similar to that proposed for low-density water in the bulk at $268 \mathrm{~K}$. For this reason our simulations lead us to conclude that the clay-water interface is longer range than the oscillations in the interlayer density profiles. The majority of the sodium counterions are fully hydrated by six water molecules. These hydrated complexes lie in contact with the solid surface (as so-called outer-sphere complexes). However, we also observe the vestiges of a diffuse layer. Diffusion is anisotropic, and is more rapid in the plane of the clay sheets than normal to the clay sheets. The former approaches the bulk value.

\section{ACKNOWLEDGMENT}

We thank the U.K. Natural and Environmental Research Council (NERC) for financial support.

${ }^{1} \mathrm{~J}$. N. Israelachvili, Intermolecular and Surface Forces (Academic, London, 1992).

${ }^{2}$ R. Guidelli and W. Schmickler, Electrochim. Acta 45, 2317 (2000).

${ }^{3}$ J. N. Israelachvili and H. Wennerstrom, Nature (London) 379, 219 (1996),

${ }^{4}$ I. Benjamin, Chem. Rev. 96, 1449 (1996).

${ }^{5}$ J. Swenson, M. V. Smalley, R. K. Thomas, R. J. Crawford, and L. F. Braganza, Langmuir 13, 6654 (1997).

${ }^{6}$ G. D. Williams, A. K. Soper, N. T. Skipper, and M. V. Smalley, J. Phys. Chem. B 102, 8945 (1998).

${ }^{7}$ R. Fenter, L. Cheng, S. Rihs, M. Machesky, M. J. Bedzyk, and N. C. Sturchio, J. Colloid Interface Sci. 225, 154 (2000).

${ }^{8}$ E. Spohr, Chem. Phys. Lett. 207, 214 (1993).

${ }^{9}$ D. A. Rose and I. Benjamin, J. Chem. Phys. 98, 2283 (1993).

${ }^{10}$ X. Xia, L. Perera, U. Essmann, and M. L. Berkowitz, Surf. Sci. 335, 401 (1995).

${ }^{11}$ E. Spohr, Electrochim. Acta 44, 1697 (1999).

${ }^{12}$ A. Delville, J. Phys. Chem. 97, 9703 (1993).

${ }^{13}$ N. T. Skipper, Miner. Mag. 62, 657 (1998).

${ }^{14}$ G. Sposito, N. T. Skipper, R. Sutton, S-H. Park, A. K. Soper, and J. A. Greathouse, Proc. Natl. Acad. Sci. U.S.A. 96, 3358 (1999).

${ }^{15}$ G. Sposito, The Chemistry of Soils (Oxford, New York, 1989).

${ }^{16}$ A. C. D. Newman, Chemistry of Clays and Clay Minerals (Mineralogical Society, London, 1987)

${ }^{17}$ E. S. Boek, P. V. Coveney, and N. T. Skipper, J. Am. Chem. Soc. 117, 12608 (1995).

${ }^{18}$ W. L. Jorgensen, J. Chandrasekhar, J. D. Madura, R. W. Impey, and M. L. Klein, J. Chem. Phys. 79, 926 (1983).

${ }^{19}$ J. Chandrasekhar, D. C. Spellmeyer, and W. L. Jorgensen, J. Am. Chem. Soc. 106, 903 (1984).

${ }^{20}$ M. W. Mahoney and W. L. Jorgensen, J. Chem. Phys. 112, 8910 (2000).

${ }^{21}$ N. T. Skipper, G. Sposito, and F-R. Chang, Clays Clay Miner. 43, 285 (1995).

${ }^{22}$ K. Refson, Comput. Phys. Commun. 3, 310 (2000).

${ }^{23}$ T. Sato, T. Watanabe, and R. Otsuka, Clays Clay Miner. 40, 103 (1992).

${ }^{24}$ H. Ohtaki and T. Radnai, Chem. Rev. 93, 1157 (1993).

${ }^{25}$ A. K. Soper and M. A. Ricci, Phys. Rev. Lett. 84, 2881 (2000).

${ }^{26}$ D. H. Powell, K. Tongkhao, S. J. Kennedy, and P. G. Slade, Clays Clay Miner. 45, 290 (1997).

${ }^{27}$ F-R. Chang, N. T. Skipper, and G. Sposito, Langmuir 11, 2734 (1995). 